\title{
Gathering and processing energy consumption data from public educational buildings over IPv6
}

\author{
Kostas Koumoutsos ${ }^{1,2}$, Aristotelis Kretsis ${ }^{1,2^{*}}$, Panagiotis Kokkinos ${ }^{1,2}$, Emmanouel A Varvarigos ${ }^{1,2}$, \\ Vassilis Nikolopoulos ${ }^{3}$, Eirini Gkioxi ${ }^{3}$ and Anastatios Zafeiropoulos ${ }^{4}$
}

\begin{abstract}
Background: Reducing energy consumption and $\mathrm{CO} 2$ emissions in order to address climate change requires behavioral changes by the citizens, who will have to adopt more environmentally friendly and energy-saving practices.

Methods: We present a system and corresponding practices for gathering energy consumption data from public school buildings over the Internet, processing them to identify hidden correlations and produce actionable advice and presenting the results in real-time to its occupants over the Web. We describe the metering infrastructure installed at schools for energy consumption monitoring and the related actions carried out in order to motivate local school communities towards an environmentally friendly behavior. The introduction of IPv6 was found to be a key enabling technology for setting up such a system in a simple, secure, and efficient way.

Results: The proposed system and processes are put under test in a pilot installation composed of about 50 IPv6-enabled schools of the Greek School Network (GSN). These are also combined with appropriate educational and social engagement tools. In this way, energy consumption, operating costs, and greenhouse effects can be reduced in the area of educational and public administration buildings in general. Thus, people become almost instantly aware of the energy and environmental implications of their actions, which motivates them towards behavioral changes and the adoption of environmentally friendly practices.
\end{abstract}

Conclusions: The pilot provides a good example of collaboration between the ICT sector, the smart building and automation vendors, and the public authorities.

Keywords: Smart energy meters; Energy information system; Advance energy analytics; IPv6; School network

\section{Background}

Reducing energy consumption and $\mathrm{CO}_{2}$ emissions in order to address climate change requires behavioral changes by the citizens, who will have to adopt more environmentally friendly and energy-saving practices. As buildings account for about $40 \%$ of total energy consumption and emissions [1,2], improving the energy behavior of their occupants is a key factor for achieving the desired climate and energy objectives. The first, but necessary and important, step in this direction is raising the energy awareness of the buildings' occupants by providing them immediate feedback on the energy implications of their actions (e.g., when turning on the lights or some equipment), in the same way that the speed measurement equipment present in a car gives the driver immediate

\footnotetext{
* Correspondence: akretsis@ceid.upatras.gr

'Department of Computer Engineering and Informatics, University of Patras, Patras, Greece

${ }^{2}$ Computer Technology Institute "Diophantus", Patras, Greece

Full list of author information is available at the end of the article
}

feedback on the implications of his actions while driving (e.g., when stepping on the gas pedal).

Energy awareness is even more important when it refers to pupils, as behavioral changes in them are more long lasting, easier to accomplish, and have larger cumulative impact. The application of behavioral change theories in the field of energy consumption supports criticism of a too narrow focus on individual behavior and the need to broadening to include social interaction $[3,4]$. So, intervention programs aimed at the change of energy consumption patterns need to take into account that behavioral change is best achieved and maintained if supported by tailored information and changes in context, for example, through supportive social networks.

It is evident that energy monitoring systems are important for energy awareness (e.g., in manufacturing companies [5], schools [6], households [7, 8], universities [9], etc.). Studies have shown that by simply monitoring one's energy consumption, the savings on the electric bills can be 5 to $15 \%$. However, Van Dam et al. [8]

\section{它}


found that the introduction of energy monitors in buildings results in only a temporary decrease in the level of energy savings made by occupants, which could not be sustained after a period of only a few months. This is attributed to a lack of habit formation as well as poor design with overly complex interfaces. The authors showed that where people adopt a regular habit of looking at energy monitors on a daily basis, they exhibit larger savings over time compared with others. An implication is to question the notion of mass-produced "one-size-fits-all" home energy monitors and whether solely technological solutions (such as energy monitors) actually achieve the desired results. It also suggests that a deeper understanding of the relationship between the user and these systems is needed. Darby [10] also discusses the level of "affordance" that smart meters offer, i.e., their usability and effectiveness and points out that: "Taking control away from the customer cannot be relied upon to improve the situation: it may actually entrench and legitimize high-demand practices, disengaging customers from any need to consider and question them". Darby argues that effective forms of interface, feedback, narrative, and support need to be developed to reach more diverse populations and to reduce actual consumption. The challenge is for the smart meter to prove itself in terms of developing a useful relationship with the user, through new forms of user engagement (understanding, expectation, and behavior) enabled by new design and media approaches.

Today, the many recent advances in energy metering technology and the design of more energy-efficient equipment have only partially been adopted in public and residential buildings. One of the reasons limiting their adoption is that their internetworking is often difficult to implement due to the lack of public IPv4 addresses (and the use of Network Address Translation (NAT) technologies, which complicate things) and the difficulties encountered in configuring the network of devices. IPv6 [11] is a technology that promises to meet the needs of the huge Internet growth (and the "Internet of things" vision), eliminating some of the restrictions caused by IPv4 technology. Thus, the IPv6 technology takes the role of an enabler and introduces various additional benefits such as security, effective access control, reduced management, and maintenance effort.

Most related works focus either on the behavioral and social aspects of energy monitoring [6] or on the pure technical details of related energy monitoring solutions $[12,13]$. In our work, we present a system and set of practices for gathering energy consumption data from public educational buildings over the Internet. We concentrate more on the technical aspects of developing, installing, and operating such a system and on the visual interactions with the users, while the presented energy consumption data are indicative of the value and the benefits of the system. In particular, the proposed system consists of a set of IPv6 enabled smart meters, an energy information system, a Web platform with social engagement tools for the pupils of the schools, and an IPv6 enabled network that interconnects them. The proposed system and processes are put under test in a pilot installation composed of about 50 schools of the Greek School Network (GSN) [14], implemented in the context of the Governments Enabled with IPv6 (GEN6) project [15]. IPv6 technology has already been deployed in GSN, providing a leveraging technology for enhancing existing services or providing new services to the end users, including the advanced energy services described in the present paper. The infrastructure installed makes use of cloud computing technologies, as the smart meters measure, and push the recorded energy consumption data to a scalable cloud aggregation system via a secure communication channel over the IPv6 protocol. The collected data is being stored, aggregated (if necessary), and analyzed using a meter data management (MDM). The services providing energy key performance indicators (KPIs), such as the kilowatt hours consumed and the corresponding volume of $\mathrm{CO}_{2}$ emissions, will be offered according to Software as a Service (SaaS) model by exposing Web services to third authorized partners. Data is made available through an API in javascript object notation (JSON) format.

An interactive IPv6 Web platform (http://gen6.sch.gr) is in charge of monitoring the processed energy consumption data coming from MDM. Via a real-time intuitive interface, the school community will be taught the correlation between the actions they undertake and the energy consumption and $\mathrm{CO}_{2}$ emissions of their schools, providing in this way significant motivation for behavioral changes. Moreover, the IPv6 Web-based platform will become an educational and social engagement tool for teachers and students, stimulating discussions and enabling actions within schools in order to better understand complex energy consumption data, through their interactive display using animations, graphical statistics, historical data, comparative schools' energy data, average factor, best performing school competition, etc. Results from the competition among the participating school communities will be disseminated to the public through various communication channels including the Web portal and social networks. At the final stage, the students will be given the opportunity to participate in a series of interactive workshops designed to develop their knowledge on environmental and energy issues, which will encourage them to take actions in the direction of sustainable living, energy consumption minimization, and climate change and motivate them to adopt a longterm environmentally friendly behavior, inside and outside the school. 
The proposed system and processes are implemented in the context of the GEN6 project [15] through the cooperation of the Computer Technology Institute (CTI) [16], Intelen [17], and the Greek Research and Technology Network (GRNET) [18] on the GSN [14]. The GEN6 project has an ambitious mission in setting up complementary and interoperable national and crossborder pilots across different EU States, in order to validate and catalyze the deployment of IPv6 in the existing EU government infrastructures, services, and applications. The GEN6 Greek Pilot presented in this work has already installed a set of IPv6-enabled smart meters at 50 schools for the real-time monitoring of their energy consumption over the IPv6-enabled network and has kicked off other dissemination actions aiming at motivating students to change their behavior towards an energy-efficient and environmentally friendly direction. Our initial tests have shown energy savings of more than $10 \%$ and reductions in the carbon footprint by at least $10 \%$ at the participating school buildings. Our target is that the developed system for monitoring, analyzing, and reporting energy consumption will also become an educational and social engagement tool for students.

The remaining of this paper is organized as follows. In the "Public education buildings" section, we describe the basic characteristics of the Greek public education buildings. The architecture of the system responsible for collecting and analyzing energy data from these buildings is presented in the "Proposed architecture" section. Details for the smart meters and the IPv6 connectivity, the data management system, and the Web platform are presented in the "Smart power meters and IPv6," "Meter data management," and "Web platform and social engagement" sections, respectively. The "Results and discussion" section presents the collected energy consumption data from a number of schools. In the "Conclusions" section, we conclude the paper.

\section{Methods}

\section{Public education buildings}

One of the reasons we focused our efforts on public educational buildings as opposed to general public building is that in the former case the buildings' architecture and topology are more suitable for running energy efficiency case studies. Usually, educational buildings are more complex and have a more distributed architecture with many individual facilities inside the campus (classroom space, library/resource space, indoor and external sports facilities), so that the energy consumed is much more than that of common public building. Moreover, the occupants of the educational buildings are students, whose energy consumption behavior should be easier to modify when compared to that of adults. Indeed, the goal of education is behavioral change, and the use of ICT in the formulation of effective teaching methods that tap into the mechanisms of behavioral change can be very efficient. In fact, some of the behavioral change theories, like the Social Learning Theory and Theory of Planned Behavior, were developed as attempts to improve behavior through education in schools. Several works that consider energy consumption issues consider schools as the primary use case $[6,19]$.

In numbers, today in Greece, there are around 3600 secondary education units and 12,000 primary education units, hosting more than 800,000 students and 100,000 teachers [20]. Public education buildings in Greece are distributed over the whole country, with varying student population sizes per building, varying floor area, and varying weather conditions. The mere energy consumption of a building is not a good measure of energy efficiency, as it has to be adjusted to account for these factors. One of the most objective KPIs used to quantify energy efficiency in schools is the "energy utilization index (EUI)" of a school which is measured in $\mathrm{kWh} / \mathrm{m}^{2} /$ student. Using objective and easy to compare KPIs is important in our case where we are also interested in initiating an energy efficiency improvements "competition" among pupils of different schools, and the competition will of course have to be fair to all participants.

GRNET [18] is the National Research and Education Network (NREN) provider, operating the Greek Academic network, that connects local universities and research institutions via dark fiber at speeds up to $10 \mathrm{Gbps}$, and offering to the Greek R\&E community access to the panEuropean GEANT network through $4 \times 10$ Gbps links. The Greek Schools' Network (GSN) [14] is the educational intranet of the Greek Ministry of Education that interlinks all primary and secondary education schools and respective administrative units, providing basic and advanced telematics services. GSN is a nationwide network that spans to all 51 prefectures of Greece, using GRNET as its backbone network (Fig. 1.) The majority of schools in Greece are connected to GSN using ADSL and optical technology.

GRNET and GSN are in strong cooperation in order to complete the transition from an IPv4-only network to a dual stack (IPv4/IPv6) network. The Greek School Network already supports IPv6 interconnection services and other basic services over IPv6 [21]. Such services are gradually extended in the intranets within schools (approximately 15,000 sites). The backbone network of GSN is fully IPv6-enabled including IPv6 support. On the access network, IPv6 interconnection has been activated for the ADSL users, i.e., for $95 \%$ of the schools. This has been achieved by enabling IPv6 on the radius profiles of each ADSL router connected to GSN. All 


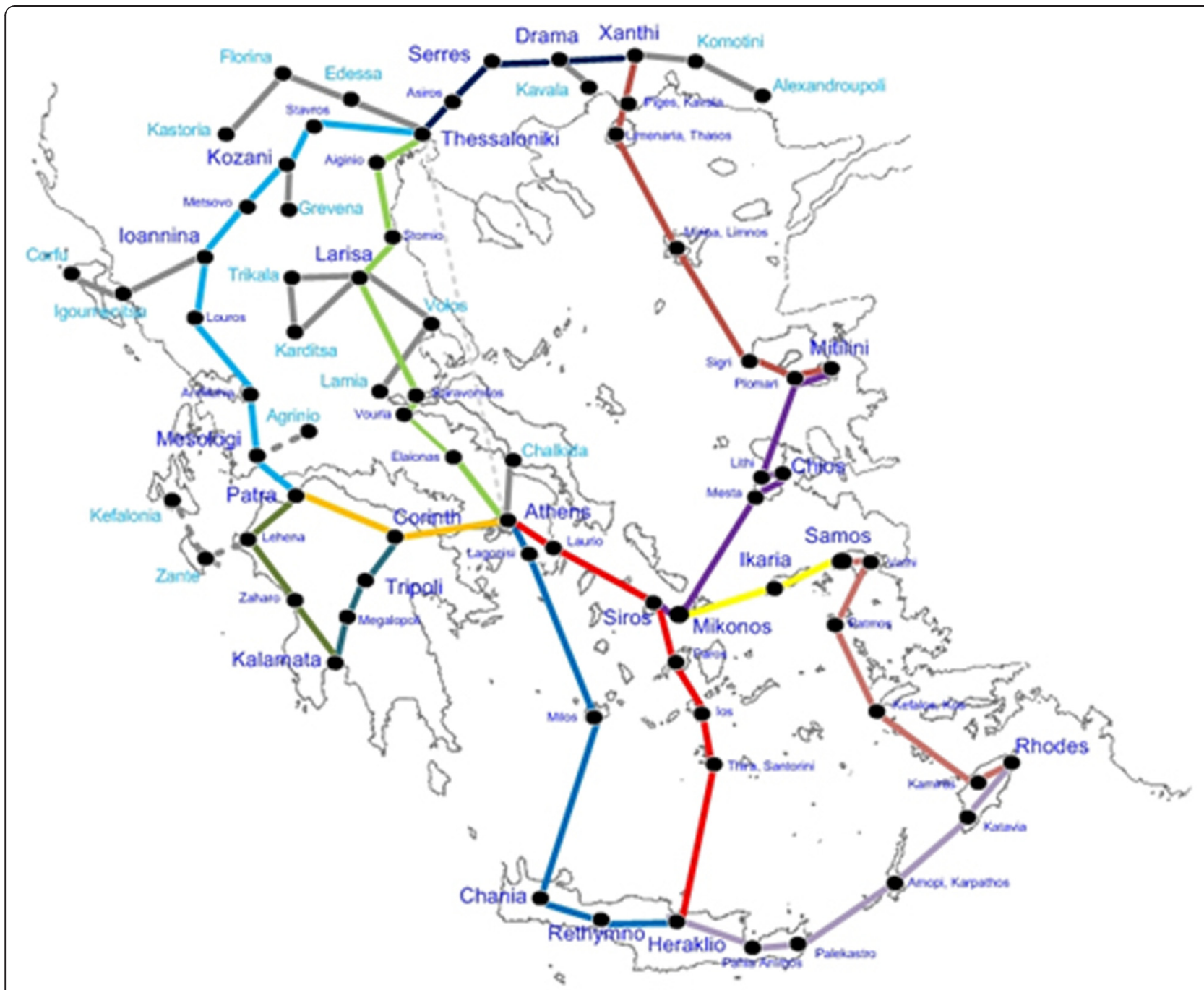

Fig. 1 GRNET and GSN network topology [24]

public education buildings are connected to the Public Power Corporation (PPC).

Developing and deploying an energy monitoring and analysis system in all public education buildings are huge tasks, considering the number of buildings and responsible personnel, the possible electrical and network peculiarities of each educational unit, and the huge amount of information that needs to be collected and analyzed. The advantages to be obtained by accomplishing this task are however also important, with the most obvious one being the energy savings and corresponding environmental benefits that could be obtained and the elimination of great costs that are spent annually to meet the schools' energy needs. The money savings can also be translated into the ability to invest in additional intervention actions for additional high performance facilities, hiring more teachers and purchasing more textbooks and computers. Beyond these bottom line benefits, schools also can realize better overall student health and better serve as centers of community life.

\section{Proposed architecture}

Advanced metering infrastructure (AMI) systems are systems that measure, collect and analyze energy usage, and communicate with metering devices such as electricity meters either on request or on a schedule. These systems include hardware, software, communications, consumer energy displays and controllers, and a MDM. AMI differs from traditional automatic meter reading (AMR) in that it enables two-way communication with the meters.

In what follows, we present such an AMI system designed for public education buildings in Greece consisting of components provided by Computer Technology Institute (CTI) [16] of the GSN [14], Intelen [17], and 
the GRNET [18]. In particular, the proposed system consists of the following components:

- energy meter

- network

- aggregator and analysis system

- user interface

Figure 2 presents the logical topology of the GEN6 Greek Pilot in schools, which consists of the aforementioned components.

The proposed system (Fig. 3) utilizes Intelen's [17] smart metering infrastructure consisting of a consumption metering device (CMD) along with its current transformer $(\mathrm{CT})$, which does not measure voltage, a transmitter, and the so-called i-box. The i-box is a smart network device that acts as a data bridge between the power meter and the local building router. Both i-box and the GSN/GRNET local router can have IPv6 global address using stateless auto-configuration method (prefix delegation). The primary internet connection in education buildings is broadband (xDSL) connection with IPv4 or IPv6 characteristics. The installed power meters collect energy consumption data that are sent over the GSN/GRNET network aggregated to Intelen's Head-End System (HES) and then to Intelen's MDM. The MDM is responsible for data acquisition and is capable for a series of services, including energy analysis, financial analysis, and demand response (signal notification, manual and automated response, response analysis, and quantification). Finally, the MDM feeds the processed information to Intelen's intercompany data exchange (IDE) system, which exposes the Web services used by the Web platform ("Web platform and social engagement" section).

\section{Smart power meters and IPv6}

The smart metering infrastructure is capable of offering a series of services, including the following:

- It enables real-time or near real-time registration of electricity use.

- It provides local and remote access to the meter (on demand).

- It performs basic calculations in order to translate the raw data that are collected by the specific CMD, to information that can be handled by centralized (cloud) infrastructure.

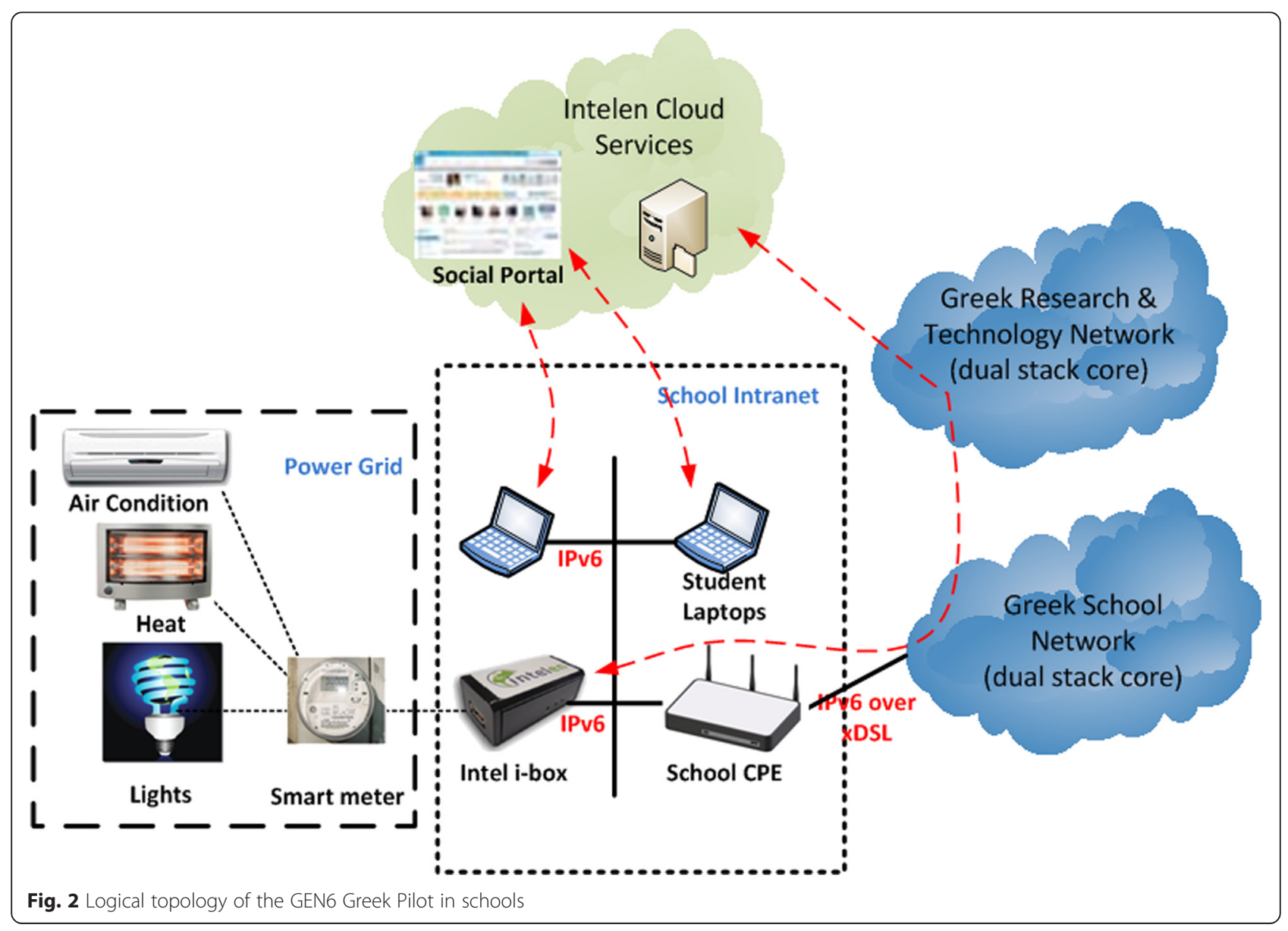




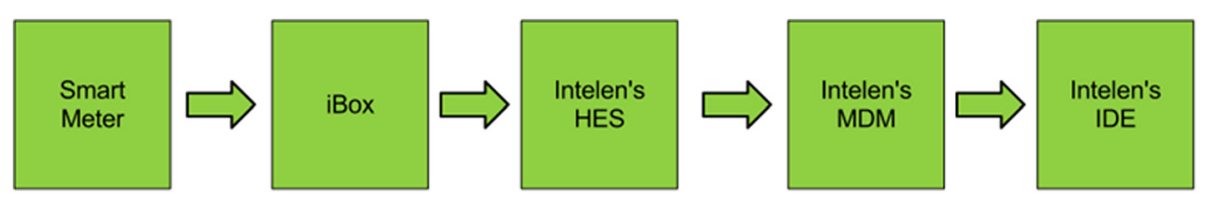

Fig. 3 The data flow from a smart meter to Intelen's intercompany data exchange (IDE) system

- It acts as memory storage (buffer) so that data are not lost in case of a network or other error but are stored (up to the size of the respective embedded storage device) for transmission when network connectivity is re-established.

- It extracts the appropriate KPIs from the raw data received by the CMD.

- It performs error handling concerning the CMD, the connectivity of the system.

- It receives instructions for extra KPIs from Intelen's MDM.

The proposed system requires not only the installation of smart energy meters but also of additional sensor nodes (e.g., temperature and humidity sensors, meteorological stations, etc.). These nodes have to operate in a reliable way, be able to recover from failures and instant networking problems, and operate for a large time period (minimum energy consumption). The deployed nodes are based on the 6LoWPAN standard (IPv6 over low power wireless personal area networks) that allows IPv6 packets to be carried efficiently within small link layer frames such as those used by IEEE 802.15.4 achieving in this way lower energy consumption. The energy efficiency of 6LoWPAN is also combined with the support of advanced ad-hoc routing, autonomic networking, and infrastructure bootstrapping characteristics. Self-* functionalities (e.g., self-configuration, self-healing, selfmanagement) can be designed and implemented over IPv6 permitting the deployment of advanced communication schemes among the sensor networking devices and reducing the administration overhead for handling faults or alarms. Based on the addressing scheme applied, each device is able to recognize its neighboring devices and an ad-hoc network may be established.

Intelen's smart meters are fully IPv6 enabled and can be assigned a global IPv6 address for their connection to the GSN/GRNET network. IPv6 is as a necessary technology for the reliable, efficient, and scalable implementation of the proposed energy metering system. Even if the major force for the transition to IPv6 is related to the absence of public addresses in IPv4, there are several IPv6 protocol characteristics that facilitate the deployment of advanced networking services, especially in the sensor networking world, and were found to be particularly useful in our proposed system. In particular, the IPv6 technology takes the role of a "green" enabler and raises various benefits such as the following:

- Reduced management and maintenance effort due to end-to-end communication and management of the smart sensors. The end-to-end communication scheme facilitates the proper management/ configuration of the devices, the installation of software updates, and the monitoring of the infrastructure.

- Better policy schemes over heterogeneous networks are made possible; for example, nodes of various on-site communications systems can be provided with unique public IPv6 addresses to avoid conflicting use of private IPv4 addressing; centralized communication initiation and management can be provided and services can be directly connected as all nodes across different on-site networks can have globally unique public IP addresses.

- Advanced security features to the schools intranets are enabled; for example, transparent end-to-end security without complex NAT traversal mechanisms, fine-grained security policies, and filtering rules can be applied based on unique end system addressing scheme.

- Advanced auto-configuration features (e.g., IPv6 stateless auto-configuration, bootstrapping of the infrastructure) and ad-hoc routing are made possible.

- Quality of Service (QoS) can be supported in local and global network environment.

- Multicast transmission features are enabled.

- Facilities are provided to deploy new services without NAT-related limitations and requirements for application gateway implementation (e.g., exchange of sensor data, services for situation monitoring, etc.).

\section{Meter data management}

A meter data management (MDM) system stores, analyzes, and displays building energy data. At a minimum, MDM provides hourly whole-building electric data that 
are Web accessible, with analytical and graphical capabilities. The MDM system was extended in order to meet the envisioned system's requirements and is used from the project Web portal in order to retrieve data from electrical energy meters. Energy consumption data from energy meters are being collected through the i-box and, every 15 min (default value), are sent to the MDM system.

The MDM system comprises of the following:

- Specific middleware for data acquisition, data aggregation, data structuring, and transformation, so as to feed all functionalities and services in a generic way.

- Functionalities and services for data validation, integration/storage, consolidation, and access.

- Generic applications that can be realized in a modular way through the invocation/combination/ orchestration of functionalities and/or services. These applications are still generic but form the basis for those offered to clients, either through parameterization or through client specific instantiation.

In the developed MDM system, energy data are processed and enriched with other school characteristics and data, such as school area, school population, etc. Data analysis is one of the most important parts of the MDM system and is also the system responsible for all calculations and data-mining algorithms, either in batch mode or in real-time. Data mining algorithms and techniques that are used by the MDM system allow users to analyze data from many different angles, categorize it, and summarize the relationships identified. In essence, data mining is the process of finding the correlations or patterns among dozens of fields in the existing large relational energy database. A recursive algorithm is executed in order to analyze the results through time and correlate them with other external variables (temp, humidity, etc.). The process of finding hidden information from data has different definitions, including knowledge extraction, information discovery, data mining, and the more proper knowledge discovering in energy database. In particular, data mining includes other activities, such as data selection, checking, cleaning, preparation, pattern presentation, and knowledge refinement and visualization. In the MDM system, a load profiling method is being applied and a specific data-mining technique on agent grid clustering is used for the processing of the data. The objective of this design is to determine the best techniques to be used, in order to reach the energy-saving target that has been set.

For effective load profiling and data mining, the following steps were followed:
- Set specific energy key performance indicators (KPI) from the metering grid and the load curve mathematics.

- Categorize and compute specific objective functions, comprised of specific KPIs.

- Perform a weighted version of the recursive analytics algorithms, based on a distributed agent structure, routed on the cloud.

- Store the numerical values in matrices, called energy relevance matrices (ERM), and use numerical linear algebra to find correlations and hidden patterns.

- Use the results of the energy relevance matrix analysis together with the output of the agent results vector in order to identify cross-hidden correlations, periodicity, and peak trends on the energy load curves.

- The proposed approach, regarding the application of game mechanics and behavioral engagement to the students, will enhance their awareness and push them towards a change in their behavior that will save energy. The IPv6-based system will analyze the real-time energy consumption and various dynamic statistical indices and metrics. Special algorithms and game mechanics/scenarios will engage the student in a continuous game by offering comparison tables and personalized incentives to save energy.

Energy data become available through APIs in JSON, XML, plain text, HTML, and CSV formats. The most appropriate format is JSON due to its flexibility and the smaller size of its files. The available API methods include daily data in 1-hour intervals, monthly data in 1 -day intervals, and yearly data in 1-month intervals.

\section{Web platform and social engagement}

Citizens' behavior is an important factor that determines the overall energy consumption of buildings and may be improved through energy awareness. An energy consumer who is "energy-aware" and is able to know in real-time the energy consumption that his/her behavior is causing and compare it to that of other similar users is more likely to initiate changes and adopt a more environmentally friendly behavior. The term "energy awareness" implies also the training of a user in terms of understanding the contribution to the total energy consumption caused by his/her use of appliances and helping him/her define specific actions that could lower the energy consumption in a quantifiable way.

We have developed an interactive Web platform [22] that is in charge of presenting the processed energy consumption data coming from the MDM system. In order to achieve this and enable the students' community better understand complex energy data, the display of the data in the students' portal has been enriched 


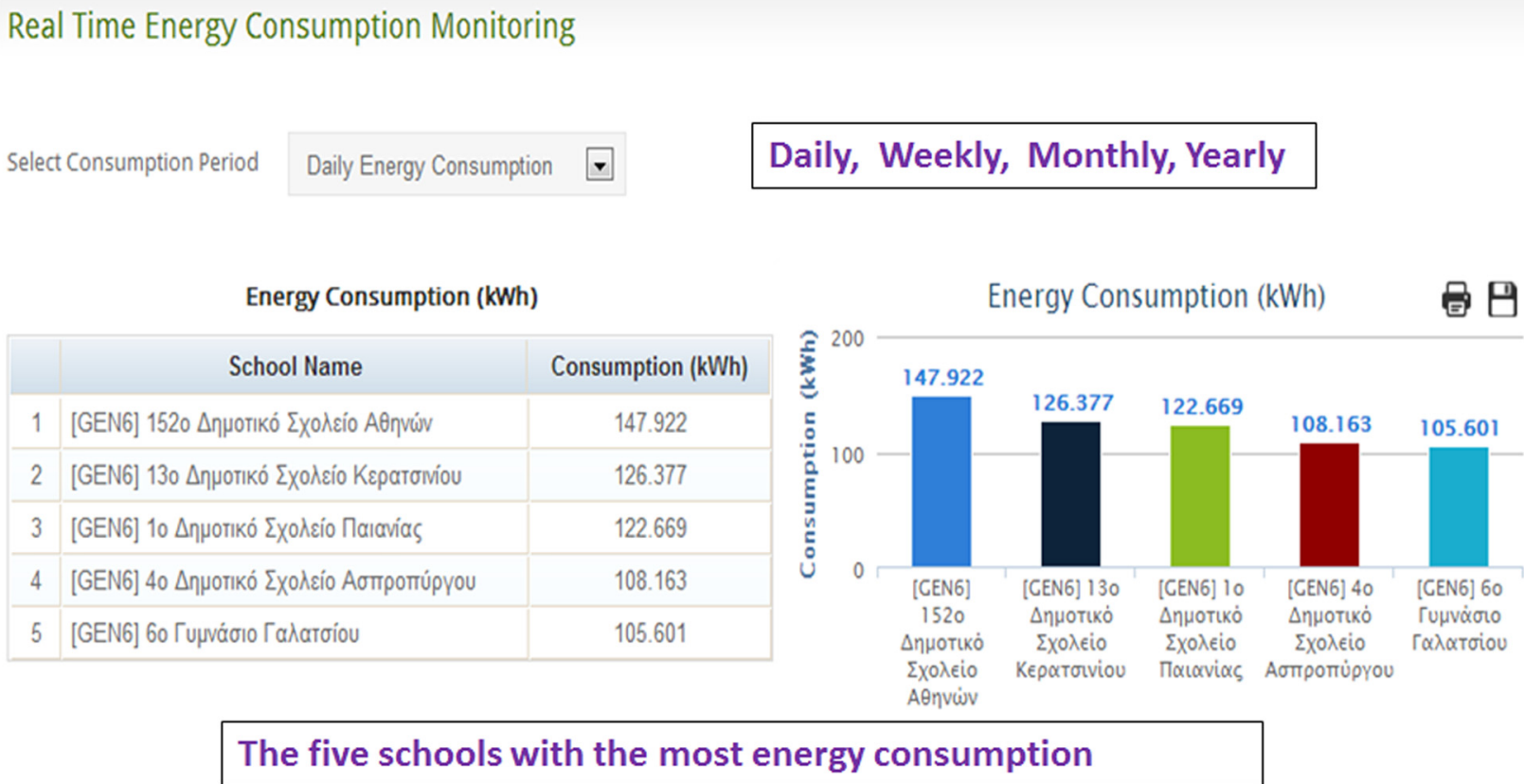

\begin{tabular}{|c|c|c|}
\hline & School Name & Consumption (kWh) \\
\hline 1 & 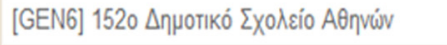 & 147.922 \\
\hline 2 & 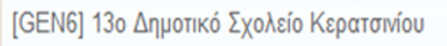 & 126.377 \\
\hline 3 & 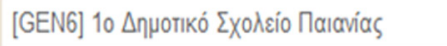 & 122.669 \\
\hline 4 & 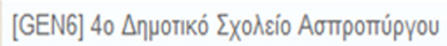 & 108.163 \\
\hline 5 & 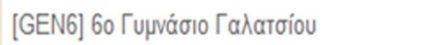 & 105.601 \\
\hline
\end{tabular}

\section{The five schools with the most energy consumption}

Fig. 4 The real-time energy consumption of schools

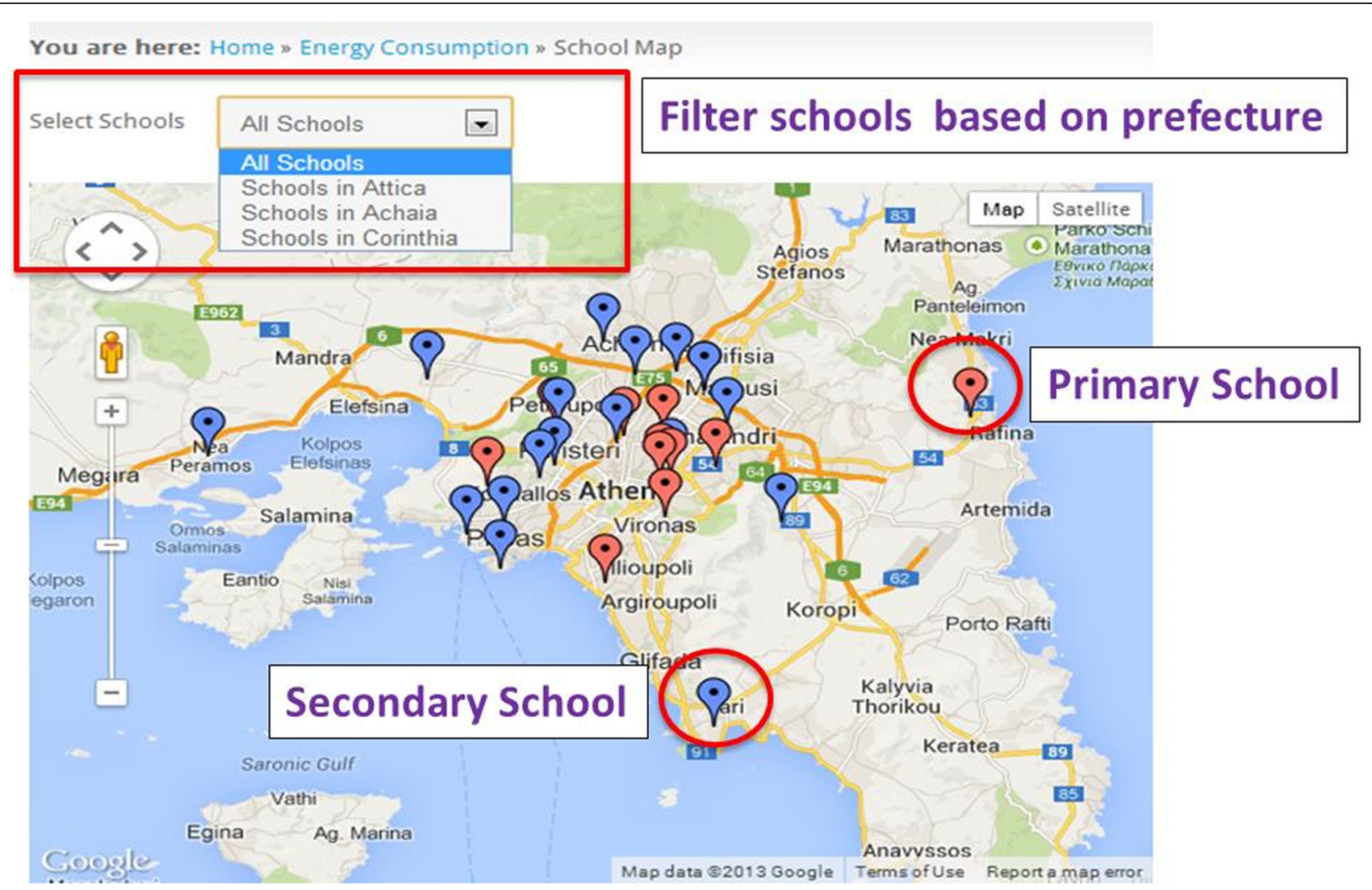

Fig. 5 Geospatial map of the measured schools 
Table 1 Energy savings

\begin{tabular}{ll}
\hline School name & Energy saving (\%) \\
\hline Attiki-14th Primary School Acharnon & 22.79 \\
Attiki-10 Primary School Varkizas & 16.98 \\
Attiki-152nd Primary School of Athens & 19.42 \\
Attiki-7th Primary School Transformation & 27.00 \\
Attiki-60 Primary School Upper Liosion & 22.66 \\
Attiki-1st Gymnasium of Alimos & 35.00 \\
Attiki-6th Gymnasium Galatsi & 35.57 \\
Attiki-49th High School of Athens & 39.18 \\
Attiki-410 Primary School of Peristeri & 19.50 \\
Attiki-1st Primary School Peanias & 33.49 \\
\hline
\end{tabular}

with animations, graphical statistics, historical data, comparative school energy data, average factor, information on the best performing school, etc.

For instance, Fig. 4 presents the real-time energy consumption of schools as it is shown in the portal [22]. The schools' energy consumption information is made more directly comparable by accounting for the number of students and the area of the school. Historical data regarding the energy consumption of schools are also available. The Web platform also associates geoinformation with schools energy profile (Fig. 5).

In general, the Web platform illustrates to the students the correlation between their actions and energy consumption $/ \mathrm{CO}_{2}$ emissions of their school, targeting to become an educational and social engagement tool for students. This platform will be used to motivate a kind of competition among schools regarding which of them will achieve the most significant energy savings in a given time period. It can also be used to stimulate discussions and actions within schools related to energy consumption and its environmental implications.

We also plan to use social media to promote energy efficiency and sustainability campaigns not only to the school community but also to the Greek public sector in general. By using social competitions, social group benchmarking, and social interfacing, the students will

Table 2 Special characteristics

\begin{tabular}{ll}
\hline No. of Buildings & 10 \\
Av. school area $\left(\mathrm{m}^{2}\right)$ & $\sim 1800 \mathrm{~m}^{2}$ \\
Av. student population & $\sim 257$ \\
& students \\
Av. energy per student/week consumption before smart & $2.5 \mathrm{kWh} /$ \\
energy meters & student \\
Av. energy per student/week consumption after smart & $1.6 \mathrm{kWh} /$ \\
energy meters & student \\
Av. energy savings/week & $27.16 \%$ \\
\hline
\end{tabular}

interact with the service in real-time (as playing a social game with real energy data), and the service will offer adaptive incentives to the student, according to their social profile and their habits. The social media campaign program will be reinforced by hands-on workshops that will engage students on issues of sustainability and empower them to make further behavioral changes. School energy competitions will create interaction among students and create additional "social media traffic."

\section{Results and discussion Pilot setup}

An important initial decision that had to be made and would influence the success and the impact of the Greek pilot was the selection of the participating schools. The selection was based on the location of the schools and their detailed characteristics:

- Location of the schools: we decided that the selected schools will be located across three adjacent prefectures (namely, Achaia, Korinthia, and Attiki) within the Greek territory. The selected prefectures present sufficiently diverse geological and climate conditions [23]. Variations in energy consumption can be monitored without, however, affecting the impact on the achieved energy savings in the pilot, which will be based on a percentage basis.

- Detailed school characteristics: GSN organized a survey to take place among the schools. The survey that was completed at the end of March 2012 collected information regarding the characteristics of the schools and the school buildings, the students' and teachers' populations, and their degree of interest in environmental issues, including existing or past activities on energy efficiency and related issues. Some important parameters of interest are the following: (1) the willingness of the school to participate in the pilot, (2) the existence or lack of broadband connectivity, (3) type of school (primary or secondary education), and the specific grades it includes; students at the last grades of primary education and the first grades of secondary education would be preferable as participants in the pilot's activities, (4) the number of students in the school (we prefer schools with a relatively high student population), (5) the availability or lack of local technical support staff, (6) any student activities related to environmental and energy efficiency issues, (7) the total area of the school, (8) the daily electricity load, (9) the ease of accessibility of the electrical panel, (10) the proximity of the electrical panel to the school computer room, (11) the number of high consumption (over $500 \mathrm{~W}$ ) 


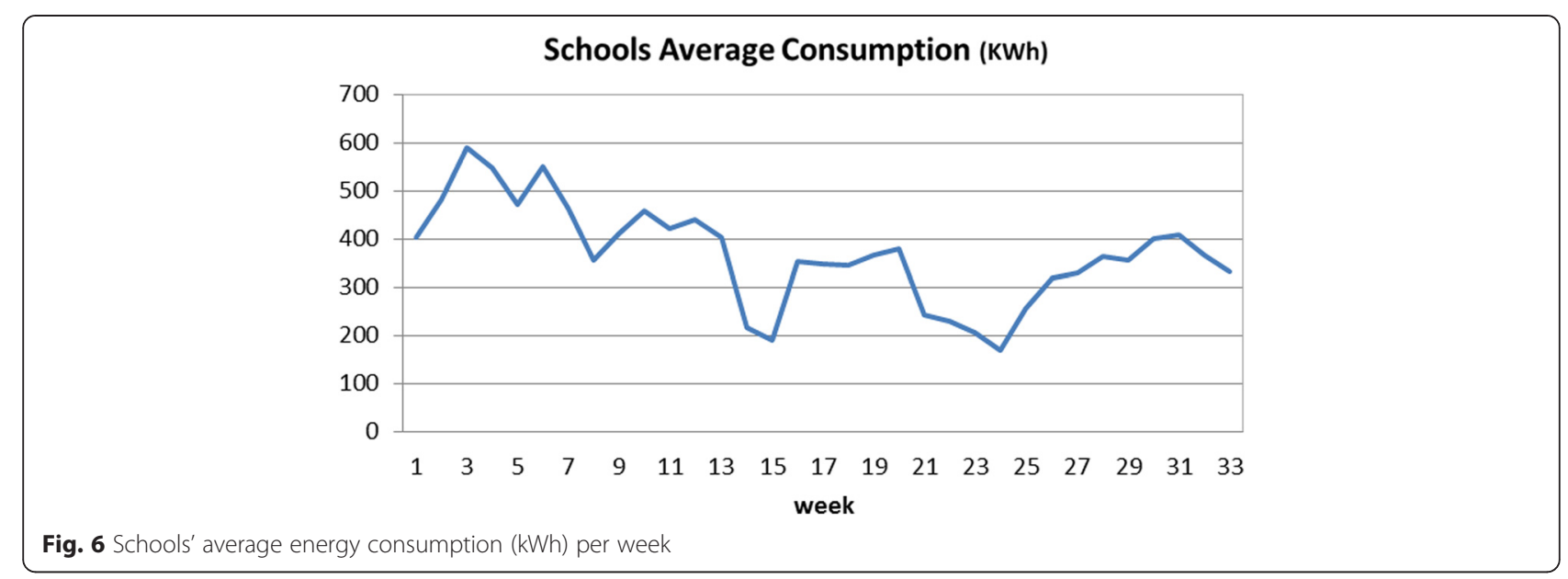

energy devices or appliances that operate at the school, (12) whether single-phase or three-phase power supply is available at the school, (13) the existence or not of a person responsible for environmental activities in the school, (14) the availability of sufficient contact information for the schools, and (15) the nominal amperes of the building's general switch.

The previous factors are actually soft requirements that have been used for the selection, and some of the schools that finally participated may not have all the desired characteristics. The final selection of the schools in the pilot has been done based on the analysis of the questionnaire's responses, interviews with interested teachers, and on-site visits that were also performed.

\section{Pilot results from 25 school buildings}

In this section, we present our initial results from the deployment of smart meters at 25 (out of the 50 schools). Actually, energy meters are currently installed in all 50 schools; however, not sufficient data from all these schools are yet available. The total energy savings for these 25 school units over a period of 33 weeks was $2945 \mathrm{kWh}$. The calculated monetary benefit is $353 €$, when the electricity school price for energy unit is 0 , $12 €$ per kWh.

The energy saving results in kilowatt hours from 10 schools after the period of 33 weeks is presented in Table 1.

These energy saving values were calculated for the 10 most "energy-efficient" schools, based on their average weekly energy consumption (over the 33-week period) in relation to the energy consumption in the first week of the evaluation. No adjustments have been made regarding other factors that may have affected the schools' consumption, such as weather (because we did not have such data available in a useable manner). Nevertheless, we believe that these results are indicative of the presented system's value and the benefits that can be obtained, even though the lack of such adjustments decrease the ability to make definite conclusions regarding the effect of the system on the students' energy behavior.

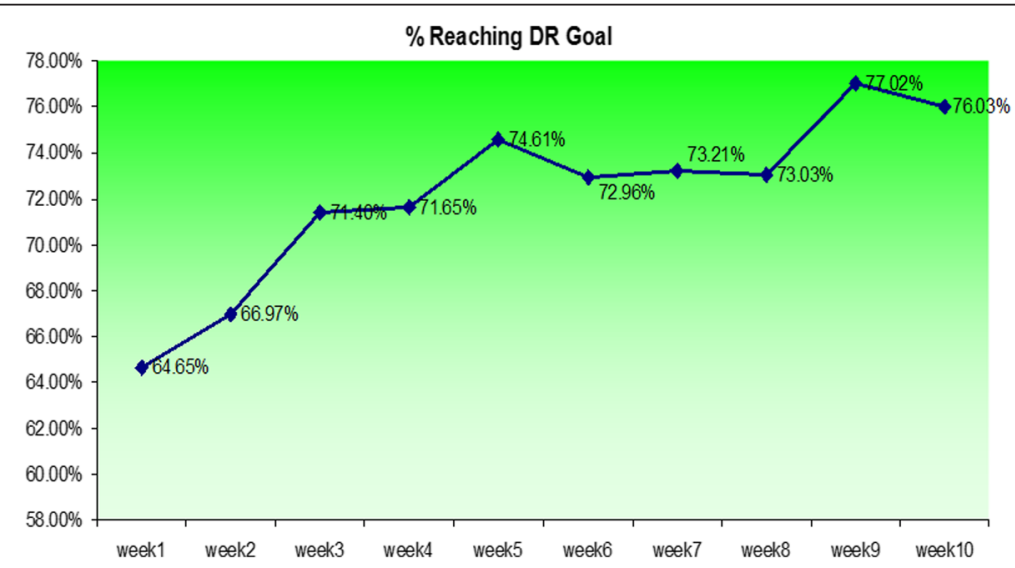

Fig. 7 Engagement efficiency in percentage to the demand response signals that were sent to the student teams 
Table 2 depicts useful details and school characteristics such as school density, school population, cumulative average energy consumption before and after the pilot installation at 10, highly "energy-efficient" schools, and finally, the cumulative average energy saving per week.

Figure 6 shows the 25 schools' average energy consumption per week. We observe that the schools consumption is decreasing, though not steadily due to variations in energy needs based weather and other reasons. The decrease cannot be with certainty attributed to the platform, as mentioned previously.

Figure 7 represents some initial results we had, following a simple demand response (DR) pilot campaign over a 10-week period, for the engagement of the students. The graph in Fig. 7 represents that the engagement efficiency as a percentage of the demand response signals that were sent to the student teams, in order to reduce their demand consumptions by specific targets in a specific timeframe (i.e., drop by $1.4 \mathrm{~kW}$ in the next $30 \mathrm{~min}$ ). The DR signals were sent using mobile SMS and email to the teams responsible. During the first weeks, the DR efficiency rose from $65 \%$ up to $74 \%$ and then was stable, fluctuating over a median. This means that from the initial DR targets, the schools were able to respond to the DR signals with some delays ( 15-20 min) and to meet their initial DR targets (drop in $\mathrm{kW}$ ) approximately by $75 \%$. Thus, the average DR efficiency reaching goal is calculated for every school for each week, as the efficiency of the transition speed ( $\mathrm{min}$ ) and the efficiency of the target demand $(\mathrm{kW})$. The dynamic average during the 10 weeks can be seen in the figure, indicating the amazing engagement progress that the students and schools have shown, towards demand response and energy efficiency targets.

\section{Conclusions}

The lack of consumers' awareness regarding their own electricity usage is a significant problem, but it also provides an opportunity for public and private stakeholders to save money and reduce $\mathrm{CO}_{2}$ emissions. The Greek School Network energy pilot aims at reducing the schools' carbon footprint by at least $10 \%$ and offer advanced real-time energy efficiency services over IPv6enabled networks. It focuses on positively affecting the students' behavior and raising energy awareness within the school communities. The installed energy meters demonstrate to participating students in real-time the energy consumption and environmental consequences of their actions, providing in this way significant motivation for behavioral changes. Given the long lifespan of most governmental buildings (including schools), their energy efficiency influences energy consumption for many years.
Our work concentrated more and presented the technical aspects of developing, installing, and deploying such an energy monitoring system in schools and on the visual interactions with the users. In this context, we recognized the importance of using the IPv6 technology for the interconnection of the smart meters with the rest of the infrastructure. Though initial results indicated an actual reduce of the energy consumption in several schools participating in the pilot, these results are not conclusive or global. Regarding overall energy consumption and reduction, it is evident that a system like the one presented in this work is only a part of the equation towards changing the energy related behavior and habits of students and people in general. Education, engagement in social interactions, and gamification are also means for achieving a sustainable energy aware and friendly attitude for all members of the society.

\section{Abbreviations}

6LoWPAN: IPV6 over low power wireless personal area networks; AMI: advanced metering infrastructure; AMR: automatic meter reading; CMD: consumption metering device; CT: current transformer; CTI: computer technology institute; DR: demand response; ERM: energy relevance matrices; EUI: energy utilization index; GEN6: governments enabled with IPv6; GRNET: Greek Research and Technology Network; GSN: Greek School Network; HES: head-end system; IDE: intercompany data exchange; KPI: key performance indicators; MDM: meter data management; NAT: network address translation; NREN: National Research and Education Network; PPC: Public Power Corporation; QoS: quality of service; SaaS: software as a service.

\section{Competing interests}

The authors declare that they have no competing interests.

\section{Authors' contributions}

KK organized the school selection procedure and drove the communication with each school authorities. Also, he was responsible for the configuration of the GSN network. KK had a lead role in the communication with the other partners for the setup of the pilot. Also, KK contributed in the writing of this paper. AK was the developer of the Web portal and contributed in the writing of this paper. PK had a role in the communication with the other partners for the setup of the pilot and in its maintenance. Also, PK contributed in the writing of this paper and in the collection of the measurements. VN participated in the development of the used advanced metering infrastructure (AMI) and also had a lead role in the installation and deployment of its components. VN provided information for the AMI that was included in this paper. EG participated in the development of the used $\mathrm{AMI}$ and also had a role in the installation and deployment of its components. EG provided information for the AMI that was included in this paper. AZ was responsible for the GRNET's network configuration and provided related information for the paper. EAV critically revised the paper and gave final approval for the paper to be published. All authors read and approved the final manuscript.

\section{Authors' information}

KK is a senior researcher in the Computer Technology Institute "Diophantus." AK is a senior researcher in the Computer Technology Institute "Diophantus." PK is a senior researcher in the Computer Technology Institute "Diophantus." EAV is a scientific director in the Computer Technology Institute

"Diophantus" and professor in the Department of Computer Engineering and Informatics, University of Patras, Greece.

$\mathrm{VN}$ is a CEO and cofounder of Intelen.

EG is a senior manager in Intelen.

AZ is a senior researcher in the Greek Research and Technology Network, Greece. 


\section{Acknowledgements}

This work was funded by the European Commission through the GEN6 project [15].

\section{Author details}

'Department of Computer Engineering and Informatics, University of Patras, Patras, Greece. ${ }^{2}$ Computer Technology Institute "Diophantus", Patras, Greece. ${ }^{3}$ Intelen Advanced Engineering Division, Nicosia, Cyprus. ${ }^{4}$ Greek Research and Technology Network, Athens, Greece.

Received: 9 January 2015 Accepted: 6 July 2015

Published online: 03 August 2015

\section{References}

1. Energy-efficient Buildings (2015) (EeB), ec.europa.eu/research/ industrial_technologies/energy-efficient-buildings_en.html, last seen May 2015

2. US energy information administration, www.eia.gov/tools/faqs/faq.cfm? $\mathrm{id}=86 \& \mathrm{t}=1$, last seen May 2015.

3. Gill T, Pegg and Allan (2010) Low-energy dwellings: the contribution of behaviours to actual performance. Build Res Inf 38:5

4. Schultz W, Nolan M, Cialdini B, Goldstein J, Griskevicius V (2007) The constructive, destructive, and reconstructive power of social norms". Psychological Science 18(5):429-434

5. Bogdanski $\mathrm{G}$ et al (2012) Energy monitoring in manufacturing companies-generating energy awareness through feedback. Leveraging technology for a sustainable world, Springer Berlin Heidelberg, pp 539-544

6. R. Kermish-Allen, K. Peterman, S. MacDonald, R. Thompson, B. Winner, "Student and teacher teams using high resolution electricity monitoring to create local change", Journal of Sustainability Education, Vol. 8, 2015

7. Hargreaves T, Michael N, Jacquelin B (2013) Keeping energy visible? Exploring how householders interact with feedback from smart energy monitors in the longer term. Energy Policy 52:126-134

8. Van Dam SS, Bakker CA, Van Hal JDM (2010) Home energy monitors: impact over the medium-term. Build Res Inf 38(5):458-469

9. Trejo-Perea M et al (2013) Development of a real time energy monitoring platform user-friendly for buildings. Procedia Technology 7:238-247

10. Darby S (2006) The effectiveness of feedback on energy consumption. A review for DEFRA of the literature on metering, billing and direct displays. Environmental Change Institute, University of Oxford, Oxford

11. Popoviciu CP, Levy-Abegnoli E, Grossetete P (2006) Deploying IPv6 networks

12. Zhao L, Ji-li Z, Ruo-bing L (2013) Development of an energy monitoring system for large public buildings. Energy Build 66:41-48

13. A. Al-Daraiseh, S. Nazaraf, and E. Eyas "An intelligent energy management system for educational buildings", International Journal of Distributed Sensor Networks, 2013. Volume 2013 (2013), Article ID 209803, 13 pages http://dx.doi.org/10.1155/2013/209803

14. Greek School Network (GSN): www.sch.gr

15. GEN6-governments enabled with IPv6, www.gen6-project.eu/.

16. CTI, Computer Technology Institute-"Diophantus", http://www.cti.gr/en/

17. Intelen: http://www.intelen.com

18. Greek Research and Technology Network-GRNET, https://www.grnet.gr/en

19. Dascalaki EG, Sermpetzoglou VG (2011) Energy performance and indoor environmental quality in Hellenic schools. Energy Build 43:718-727

20. Greek ministry of education and religious affairs archives: archive.minedu.gov.gr/el_ec_category127.htm

21. Kalogeras D, Friaças C, Ferreira JN (2005) Cookbook on deploying IPv6 in school networks

22. GEN6 Greek pilot: gen6.sch.gr/index.php/en

23. GeoKlima: http://www.geoclima.eu/node/91

24. GRNET topology: www.noc.grnet.gr/en/node/25

\section{Submit your manuscript to a SpringerOpen ${ }^{\circ}$ journal and benefit from:}

- Convenient online submission

- Rigorous peer review

- Immediate publication on acceptance

- Open access: articles freely available online

- High visibility within the field

- Retaining the copyright to your article

Submit your next manuscript at $>$ springeropen.com 